\title{
Cranial Nerves IX To XII
}

Most lesions that affect the lower cranial nerves are centered at the jugular foramen, where cranial nerves IX, X, and XI leave the calvarium (Castillo and Mukherji, 1996; Rubinstein et al., 1995). Because of the presence of the jugular vein in the same location, a strategy based on differentiating neurogenic lesions (e.g., schwannomas, meningiomas) from vascular ones (e.g., paragangliomas, high-riding jugular bulbs, aneurysms) must be employed. Cranial nerve XII lesions are usually schwannomas or secondary invasions from skull-base neoplasms (Thompson and Smoker, 1994; Karpati et al., 1998; Russo et al., 1997). This sometimes means performing MR arteriograms or venograms in addition to the standard fare of high-resolution and contrast-enhanced scans. These nerves have predictable courses and symptom complexes, which make demonstration of pertinent lesions very gratifying.

\section{IMAGING OF CRANIAL NERVES IX-XII}

Cranial nerves IX to XII are rarely affected by pathology compared with cranial nerves III, V, VII, and VIII. Nonetheless, their evaluation is challenging, since lesions of these nerves span the gamut from intracranial to extracranial sites. In the case of the vagus nerve, one may need to scan the full neck and chest to ascertain the source of the vagal symptoms. The glossopharyngeal (cranial nerve IX) and vagus nerve (cranial nerve X) are more commonly affected by diseases than the spinal accessory nerve (cranial nerve XI, which receives input from cervical nerve rootlets) and hypoglossal nerve (cranial nerve XII; Castillo and Mukherji, 1996; Chong and Fan, 1996; Ortiz and Reed, 1995). While IX to XI traverse the jugular foramen (where the jugular vein is the dominant structure, replete with its accompanying flow artifacts) cranial nerve XII exits via the smaller hypoglossal canal at the foramen magnum. Imaging of these cranial nerves requires a focused approach based on clinical symptomatology and signs. Thin slice imaging, high resolution, and contrast enhancement are essential components to this evaluation.

Table A7.4.1 lists the hardware necessary to perform the procedure, along with appropriate parameters. Standard head coil imaging is sufficient to obtain adequate scans on the intracranial portions of these cranial nerves. Anatomic imaging with high-quality fast spin echo $T_{2}$-weighted scan with the dark nerves outlined by bright cerebrospinal fluid (CSF) are required to see the portions of these nerves coursing to the jugular foramen and hypoglossal canal. Gadolinium-enhanced scans are critical to the complete evaluation of these nerves, though the evaluation may be limited to sections through the lower pons and medulla. No monitoring is required. It may be necessary to continue the evaluation into the head and neck region with a neck coil in the case of vagus nerve lesions. Particularly

Table A7.4.1 Equipment Parameters for Cranial Nerve Imaging

\begin{tabular}{ll}
\hline Coil type & Head \\
Gradient coil strength & $25 \mathrm{mT} / \mathrm{m}$ \\
Flow compensation pulse & Yes \\
Respirator & If needed \\
Oxygen & If needed \\
Motion cushions & Useful \\
Use of contrast agents & Yes \\
\hline
\end{tabular}


if a source of a vocal cord paralysis is being pursued, then a coil that covers the brainstem, the neck, and even the upper mediastinum to the aortic arch may be required. For upper vagus or glossopharyngeal or hypoglossal masses, the coverage afforded by most head coils is sufficient, provided the patient's vertex is placed at the very top of the head coil.

\section{Materials}

Normal saline $(0.9 \% \mathrm{NaCl})$, sterile

Gadolinium-based MR contrast agent (e.g., Magnevist, Omniscan, or Prohance)

\section{Set up equipment and patient}

1. Interview (screen) the patient to ensure that he or she has no contraindications such as cardiac pacemakers or other implants containing ferromagnetic materials. Also be sure to find out if the patient has any health conditions that may require the presence of special emergency equipment during the scanning procedure, or necessitate any other precautions.

Generally standard screening forms are used for all patients scanned in a magnetic resonance system.

The presence of any ferromagnetic metals may be a health hazard to the patient when he or she is inside the magnet, and will also affect the imaging. If in doubt as to the exact composition of the items, it is best to exclude patients with any metal implants; see Shellock (1996) for discussion of what implants may be safely scanned using magnetic resonance.

Patients may be accompanied into the magnet room by a friend or family member, who can sit in the room during the scan and comfort the patient as needed. This companion must be screened as well to ensure the absence of loose metal objects on the body or clothing.

2. If the procedure is a research protocol, have the patient sign any necessary consent form.

3. Have the patient remove all jewelry and change into a gown to eliminate any metal that might be found in clothing.

4. Have the patient wash off any mascara and other makeup to avoid local tissue heating and image artifacts.

5. Inform the patient about what will occur during the procedure, what he or she will experience while in the magnet, and how to behave, including the following:

a. If earphones or headphones are used to protect the ears from the loud sounds produced by the gradients, the patient will be asked to wear these, but will be able to communicate with you at any time during the imaging.

b. The patient will be given a safety squeeze-bulb or similar equipment to request assistance at any time (demonstrate how this works).

c. For good results the patient should not talk, and should avoid or minimize swallowing or other movement, during each scan-i.e., as long as the banging sounds continue. Between scans, talking and swallowing are allowed in most cases, but should be avoided when comparative positional studies are being performed; the patient will be informed when this is the case.

d. Nevertheless, the patient may call out at any time if he or she feels it necessary.

6. Help the patient mount onto the table. Either before or right after the patient lies down, set up any triggering devices or other monitoring equipment that is to be used.

7. Center the patient's head within the head coil and use the laser light to ensure symmetry. For most brain scans, placing the center landmark at the eyebrow (nasion) level allows full coverage of the relevant regions of anatomy. 
Generally the patient's head is fixed so that the head is horizontal (not tilted) and the neck and head lie along the axis of the patient table; other positions may be appropriate depending on the needs at hand.

8. If needed, place a pillow or other support under the knees to make the patient more comfortable.

9. Establish an intravenous line from which the contrast agent can be injected, and attach this line securely to the patient so that movement into or out of the magnet will not pull at the patient's arm.

It is preferable to insert the line prior to imaging and to leave the patient in the magnet, with no intervening motion, between the scans run before contrast agent injection and those run after injection.

10. Use the centering light to position the patient and put him or her into the center of the magnet.

Once this step has been performed, so long as the patient does not move on the table, the table itself can be moved and then replaced in the same position as before without jeopardizing the positioning of one scan relative to another.

11. If the patient is unable to hold still, provide an appropriate sedative.

\section{Sequence 1: Sagittal $T_{1}$-weighted spin echo scan}

Although a pilot scan can be acquired, it is not necessary here because the first scan covers almost the entire brain in a sagittal fashion. These sagittal images can then be used as localizers for the application of saturation pulses when the transverse images are acquired.

12. Run sagittal $T_{1}$-weighted scan (See Table A7.4.2).

Saturation pulses inferiorly are applied. This scan is critical to visualizing the jugular foramen and the jugular vein. Because the saturation pulses applied produce a nice flow void in the jugular foramen, one may be able to exclude a glomus jugulare with this sequence. Since the transverse scans that follow may be confusing due to turbulent flow simulating a jugular foramen mass, the sagittal scans often are the most reliable in this regard. When positioned properly, these scans may also be the ones that demonstrate carotid body tumors that may produce vagus nerve or sympathetic plexus symptoms.

\section{Sequence 2: Transverse fast spin echo (FSE) scan}

13. Run transverse fast spin echo (or turbo spin echo) $T_{2}$-weighted scans (see Table A7.4.3) through the entire brain.

Echo train length 12-32 depending on slices allowed and minimum echo spacing.

Saturation pulses are applied inferiorly and gradient moment nulling is applied. One now routinely applies frequency selective fat suppression pulses to produce a better dynamic range of contrast and to null the skull base, jugular foramen, and temporal bone fat. Acquisition time will be 3 to 4 min in length. The lower cranial nerves are well outlined in the bright CSF.

\section{Sequence 3: Transverse FLAIR scan}

14. Run transverse fluid attenuation inversion recovery (FLAIR) scan (see Table A7.4.4).

Echo train length 12-32 depending on slices allowed and minimum echo spacing. Saturation pulses inferiorly, tailored radiofrequency pulses and gradient moment nulling are applied. The slices should be through the entire brain. Acquisition time will be 4 to 5 min in length.

Demyelinating disorders may lead to lower cranial nerve symptoms. Although FLAIR does well at displaying white matter lesions in the brainstem and supratentorial compartment, recent studies have suggested that the $T_{2}$-weighted sequence remains the most reliable means of evaluating the posterior fossa white matter and cortex. 
Sequence 4: Transverse echo planar/diffusion weighted scan

15. Run transverse echo planar/diffusion weighted imaging (see Table A7.4.5).

The minimum diffusion weighting should have a " $b$ " value of at least $1000 \mathrm{sec} / \mathrm{mm}^{2}$.

These slices should be applied through the entire brain and aid in the detection of acute stroke processes.

Table A7.4.2 Sagittal $T_{1}$-Weighted Spin Echo Scan (Sequence 1) ${ }^{a}$

\begin{tabular}{ll}
\hline Patient position & Supine \\
Scan type & Spin echo \\
Imaging plane (orientation) & Sagittal \\
Central slice or volume center & Mid-brain \\
Echo time $\left(T_{\mathrm{E}}\right)$ & Minimum \\
Receiver bandwidth $(\mathrm{RBW})$ & $16 \mathrm{kHz}$ \\
Repeat time $\left(T_{\mathrm{R}}\right)$ & $400-600 \mathrm{msec}$ \\
Flip angle $(\mathrm{FA})$ & $90^{\circ}$ \\
Fields of view $\left(\mathrm{FOV}, \mathrm{FOV}_{\mathrm{y}}\right)$ & $240 \mathrm{~mm}, 240 \mathrm{~mm}$ \\
Resolution $(\Delta x, \Delta y)$ & $0.94 \mathrm{~mm}, 1.25 \mathrm{~mm}$ \\
Number of data points collected $\left(N_{\mathrm{x}}, N_{\mathrm{y}}\right)$ & 256,192 \\
Slice thickness $(\Delta z)$ & $5 \mathrm{~mm}$ \\
Number of slices & 28 \\
Slice gap & $0 \mathrm{~mm}$ \\
Number of excitations $(\mathrm{NEX})$ & 1 \\
Spatial saturation & Inferior \\
Slice series & Interleaved \\
Scan time & $\sim 2 \mathrm{~min}$ \\
\hline
\end{tabular}

${ }^{a}$ This sequence covers entire brain.

Table A7.4.3 Transverse Fast Spin Echo Scan (Sequence 2) ${ }^{a}$

\begin{tabular}{ll}
\hline Patient position & Supine \\
Scan type & Fast spin echo \\
Imaging plane (orientation) & Transverse \\
Central slice or volume center & Mid-brain \\
Echo time $\left(T_{\mathrm{E}}\right)$ & $90-120 \mathrm{msec}$ \\
Receiver bandwidth $(\mathrm{RBW})$ & $20 \mathrm{kHz}$ \\
Echo train length $(\mathrm{ETL})$ & $12-32$ \\
Repeat time $\left(T_{\mathrm{R}}\right)$ & $3000-4000 \mathrm{msec}$ \\
Flip angle $(\mathrm{FA})$ & $90^{\circ}$ \\
Fields of view $(\mathrm{FOV}, \mathrm{FOV}$ & $\mathrm{y})$ \\
Resolution $(\Delta x, \Delta y)$ & $240 \mathrm{~mm}, 240 \mathrm{~mm}$ \\
Number of data points collected $\left(N_{\mathrm{x}}, N_{\mathrm{y}}\right)$ & $0.94 \mathrm{~mm}, 1.25 \mathrm{~mm}$ \\
Slice thickness $(\Delta z)$ & 256,192 \\
Number of slices & $5 \mathrm{~mm}$ \\
Slice gap & Variable $(\sim 20)$ \\
Number of excitations $(\mathrm{NEX})$ & $0 \mathrm{~mm}$ \\
Flow compensation & 2 \\
ZIP 512 & Yes \\
Chemical saturation & Yes \\
Spatial saturation & Yes, fat \\
Slice series & Inferior \\
Scan time & Interleaved \\
\hline
\end{tabular}

${ }^{a}$ This sequence covers entire brain. 
Sequence 5: 2-D time of flight scan

16. Perform 2-D time of flight scan (see Table A7.4.6).

Because of the flow artifact associated with the jugular vein in the jugular foramen, it may be helpful to perform an MR venographic study through the posterior fossa and the skull base. This can be relegated to a 2-D time-of-flight (TOF) mode (see Table A7.4.6) where

Table A7.4.4 Transverse FLAIR Scan (Sequence 3) ${ }^{a}$

\begin{tabular}{ll}
\hline Patient position & Supine \\
Scan type & Inversion recovery fast spin echo \\
Imaging plane (orientation) & Transverse \\
Central slice or volume center & Mid-brain \\
Echo time $\left(T_{\mathrm{E}}\right)$ & $133 \mathrm{msec}$ \\
Receiver bandwidth $(\mathrm{RBW})$ & $16 \mathrm{kHz}$ \\
Echo train length $(\mathrm{ETL})$ & $12-32$ \\
Repeat time $\left(T_{\mathrm{R}}\right)$ & $8800 \mathrm{msec}$ \\
Inversion time $\left(T_{\mathrm{I}}\right)$ & $2200 \mathrm{msec}$ \\
Flip angle $(\mathrm{FA})$ & $180^{\circ}$ \\
Fields of view $(\mathrm{FOV}, \mathrm{FOV}$ & \\
Resolution $(\Delta x, \Delta y)$ & $240 \mathrm{~mm}, 240 \mathrm{~mm}$ \\
Number of data points collected $\left(N_{\mathrm{x}}, N_{\mathrm{y}}\right)$ & $0.94 \mathrm{~mm}, 0.94 \mathrm{~mm}$ \\
Slice thickness $(\Delta z)$ & 256,256 \\
Number of slices & $5 \mathrm{~mm}$ \\
Slice gap & Variable $(\sim 20)$ \\
Number of excitations $(\mathrm{NEX})$ & $0 \mathrm{~mm}$ \\
Flow compensation & 1 \\
ZIP 512 & Yes \\
Tailored RF & Yes (changes resolution to 0.47 \\
Spatial saturation & mm, $0.47 \mathrm{~mm})$ \\
Slice series & Yes \\
Scan time & Inferior \\
\hline
\end{tabular}

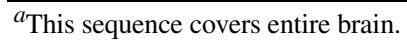

Table A7.4.5 Transverse Echo Planar/Diffusion Weighted Scan (Sequence 4) ${ }^{a}$

\begin{tabular}{ll}
\hline Patient position & $\begin{array}{l}\text { Supine } \\
\text { Ecan type }\end{array}$ \\
Echo planar/diffusion weighted \\
image \\
Imaging plane (orientation) & Transverse \\
Central slice or volume center & Mid-brain \\
Echo time $\left(T_{\mathrm{E}}\right)$ & Minimum \\
Receiver bandwidth $(\mathrm{RBW})$ & $62 \mathrm{kHz}$ \\
Repeat time $\left(T_{\mathrm{R}}\right)$ & $10,000 \mathrm{msec}$ \\
Flip angle $(\mathrm{FA})$ & $90^{\circ}$ \\
Fields of view $\left(\mathrm{FOV}, \mathrm{F}_{\mathrm{x}}, \mathrm{FOV}_{\mathrm{y}}\right)$ & $240 \mathrm{~mm}, 240 \mathrm{~mm}$ \\
Resolution $(\Delta x, \Delta y)$ & $1.88 \mathrm{~mm}, 1.88 \mathrm{~mm}$ \\
Number of data points collected $\left(N_{\mathrm{x}}, N_{\mathrm{y}}\right)$ & 128,128 \\
Slice thickness $(\Delta z)$ & $5 \mathrm{~mm}$ \\
Number of slices & Variable $(\sim 10)$ \\
Slice gap & $0 \mathrm{~mm}$ \\
Number of excitations $(\mathrm{NEX})$ & 1 \\
Slice series & Interleaved \\
Scan time & $\sim 1 \mathrm{~min}$ \\
\hline
\end{tabular}

${ }^{a}$ This sequence covers entire brain. 
Table A7.4.6 2-D Time of Flight Scan (Sequence 5) ${ }^{a}$

\begin{tabular}{ll}
\hline Patient position & Supine \\
Scan type & 2-D gradient echo \\
Imaging plane (orientation) & Coronal \\
Central slice or volume center & Skull base \\
Echo time $\left(T_{\mathrm{E}}\right)$ & Minimum \\
Receiver bandwidth $(\mathrm{RBW})$ & $16 \mathrm{kHz}$ \\
Repeat time $\left(T_{\mathrm{R}}\right)$ & $30 \mathrm{msec}$ \\
Flip angle $(\mathrm{FA})$ & $60^{\circ}$ \\
Fields of view $\left(\mathrm{FOV}, \mathrm{FOV}_{\mathrm{y}}\right)$ & $240 \mathrm{~mm}, 180 \mathrm{~mm}$ \\
Resolution $(\Delta x, \Delta y)$ & $0.47 \mathrm{~mm}, 0.70 \mathrm{~mm}$ \\
Number of data points collected $\left(N_{\mathrm{x}}, N_{\mathrm{y}}\right)$ & 512,256 \\
Slice thickness $(\Delta z)$ & $2 \mathrm{~mm}$ \\
Number of slices & Variable $(\sim 40)$ \\
Slice gap & $0 \mathrm{~mm}$ \\
Number of excitations $(\mathrm{NEX})$ & 1 \\
Flow compensation & Yes \\
Spatial saturation & Inferior \\
Slice series & Interleaved \\
Scan time & $\sim 5-6 \mathrm{~min}$ \\
\hline
\end{tabular}

\footnotetext{
${ }^{a}$ In order to suppress the arterial phase and accentuate the venogenic phase, the saturation pulse should be a "walking" also known as a "traveling" saturation pulse. The saturation pulse should move with the actual imaging slice, thereby increasing its efficacy in overall arterial suppression.
}

inferior saturation pulses are applied and downward flow is optimally demonstrated. Once again, viewing the raw data provides one with the visualization of the "opacified" vessel and the suspected "mass." Since most glomus jugulare paragangliomas grow into the jugular vein, the presence of a "filling defect" amidst the jugular vein flow would suggest this diagnosis. Lesions outside the vein may be schwannomas. If this 2-D TOF is to be utilized, it should be used prior to the administration of any contrast medium.

Another very useful imaging tool to assess the jugular vein and visualize the vessel and suspected "mass," would be the addition of a 3-D fast gradient echo pulse sequence with a double dose of contrast medium. This rapid imaging technique should give the dynamic evaluation of an arterial through venous phase of vessel filling, as well as multi-planar reconstruction. In addition, there will be an overall improved SNR (signal to noise ratio) with respect to conventional 2-D imaging techniques.

\section{Sequence 6: Transverse post-contrast $T_{1}$-weighted scan}

17. Leave the patient in the magnet, inject the contrast agent, and flush the line with 10 $\mathrm{ml}$ saline.

A dose of $0.2 \mathrm{mmol} / \mathrm{kg}$ of contrast agent is usually given.

18. Run transverse post-gadolinium $T_{1}$-weighted scan (see Table A7.4.7).

A 256 by 256 matrix through the pons, medulla and foramen magnum is used. Saturation pulses inferiorly and gradient moment nulling (flow compensation) are applied.

Pre-gadolinium scans are often not necessary and only add time. However, if in a pinch, due to the suspicion of a lesion that could be bright precontrast, simply reconstruct the sagittal $5 \mathrm{~mm}$ contiguous $T_{1}$-weighted images from the first sequence into a transverse plane and correlate the two. For some, it may be easier to perform transverse pre-contrast studies and eliminate this potential pitfall. If this is done, the parameters should not change from pre-to post-contrast studies, other than single average scans for pre-contrast series. 
Table A7.4.7 Transverse Post-Contrast $T_{1}$-Weighted Scan (Sequence 6)

\begin{tabular}{ll} 
Patient position & Supine \\
Scan type & Spin echo \\
Imaging plane (orientation) & Transverse \\
Central slice or volume center & Mid-brain \\
Echo time $\left(T_{\mathrm{E}}\right)$ & Minimum \\
Receiver bandwidth $(\mathrm{RBW})$ & $16 \mathrm{kHz}$ \\
Repeat time $\left(T_{\mathrm{R}}\right)$ & $400-600 \mathrm{msec}$ \\
Flip angle $(\mathrm{FA})$ & $90^{\circ}$ \\
Fields of view $(\mathrm{FOV}, \mathrm{x}, \mathrm{FOV}$ & $\mathrm{y})$ \\
Resolution $(\Delta x, \Delta y)$ & $180 \mathrm{~mm}, 180 \mathrm{~mm}$ \\
Number of data points collected $\left(N_{\mathrm{x}}, N_{\mathrm{y}}\right)$ & $0.70 \mathrm{~mm}, 0.70 \mathrm{~mm}$ \\
Slice thickness $(\Delta z)$ & 256,256 \\
Number of slices & $3 \mathrm{~mm}$ \\
Slice gap & Variable $(\sim 20)$ \\
Number of excitations $(\mathrm{NEX})$ & $0 \mathrm{~mm}$ \\
Flow compensation & $2-3$ \\
Spatial saturation & Yes \\
Slice series & Inferior \\
Scan time & Interleaved \\
\hline
\end{tabular}

Table A7.4.8 Coronal Post-Contrast $T_{1}$-Weighted Scan (Sequence 7)

\begin{tabular}{ll}
\hline Patient position & Supine \\
Scan type & Fast spin echo \\
Imaging plane (orientation) & Coronal \\
Central slice or volume center & Mid-brain \\
Echo time $\left(T_{\mathrm{E}}\right)$ & Minimum \\
Receiver bandwidth $(\mathrm{RBW})$ & $16 \mathrm{kHz}$ \\
Echo train length $(\mathrm{ETL})$ & $\leq 4$ \\
Repeat time $\left(T_{\mathrm{R}}\right)$ & $400-600 \mathrm{msec}$ \\
Flip angle $(\mathrm{FA})$ & $90^{\circ}$ \\
Fields of view $\left(\mathrm{FOV}, \mathrm{x}, \mathrm{FOV}_{\mathrm{y}}\right)$ & $180 \mathrm{~mm}, 180 \mathrm{~mm}$ \\
Resolution $(\Delta x, \Delta y)$ & $0.70 \mathrm{~mm}, 0.70 \mathrm{~mm}$ \\
Number of data points collected $\left(N_{\mathrm{x}}, N_{\mathrm{y}}\right)$ & 256,256 \\
Slice thickness $(\Delta z)$ & $3 \mathrm{~mm}$ \\
Number of slices & Variable \\
Slice gap & $0 \mathrm{~mm}$ \\
Number of excitations $(\mathrm{NEX})$ & 2 \\
Flow compensation & Yes \\
ZIP 512 & Yes (changes resolution to 0.35 \\
& mm, $0.35 \mathrm{~mm})$ \\
Chemical saturation & Yes, fat \\
Spatial saturation & Inferior \\
Slice series & Interleaved \\
Scan time & $\sim 2-4$ min \\
\hline
\end{tabular}


Table A7.4.9 $\quad T_{2}{ }^{*}$-Weighted Gradient Echo Scan (Sequence 9)

\begin{tabular}{ll}
\hline Patient position & Supine \\
Scan type & Gradient echo \\
Imaging plane (orientation) & Transverse \\
Central slice or volume center & Mid-brain \\
Echo time $\left(T_{\mathrm{E}}\right)$ & $20 \mathrm{msec}$ \\
Receiver bandwidth $(\mathrm{RBW})$ & $16 \mathrm{kHz}$ \\
Repeat time $\left(T_{\mathrm{R}}\right)$ & $500 \mathrm{msec}$ \\
Flip angle $(\mathrm{FA})$ & $20^{\circ}$ \\
Fields of view $\left(\mathrm{FOV}, \mathrm{FOV}_{\mathrm{y}}\right)$ & $240 \mathrm{~mm}, 180 \mathrm{~mm}$ \\
Resolution $(\Delta x, \Delta y)$ & $0.94 \mathrm{~mm}, 0.94 \mathrm{~mm}$ \\
Number of data points collected $\left(N_{\mathrm{x}}, N_{\mathrm{y}}\right)$ & 256,192 \\
Slice thickness $(\Delta z)$ & $5 \mathrm{~mm}$ \\
Number of slices & Variable $(\sim 20)$ \\
Slice gap & $0 \mathrm{~mm}$ \\
Number of excitations $(\mathrm{NEX})$ & 1 \\
Spatial saturation & Inferior \\
Slice series & Interleaved \\
Scan time & $\sim 3 \mathrm{~min}$ \\
\hline
\end{tabular}

Sequence 7: Coronal post-contrast $T_{1}$-weighted scan

19. Run coronal post-gadolinium fast spin echo $T_{1}$-weighted scan (see Table A7.4.8).

Frequency selective fat suppression pulses are applied. Saturation pulses inferiorly placed in conjunction with gradient moment nulling (flow compensation) are encouraged. The slices should include the whole of the temporal bone and brainstem. This $T_{1}$-weighted sequence is being performed as a fast spin echo $T_{1}$-weighted scan, so be sure to utilize an echo train length not to exceed 4 and make an attempt to ZIP the display matrix to 512 by 512. Acquisition time will be 2 to 4 min in length.

\section{Sequence 9: $T_{2}{ }^{*}$-weighted gradient echo scan for hemosiderosis}

20. If hemosiderosis is contemplated, perform a $T_{2}{ }^{*}$-weighted gradient echo scan through the posterior fossa (see Table A7.4.9).

This allows better detection of blood products than fast spin echo techniques (Pribitkin et al., 1994).

ALTERNATE PROTOCOL

Cranial Nerves IX To XII

\section{IMAGING OF CRANIAL NERVES IX TO XII WHERE NON-NEOPLASTIC LESIONS ARE SUSPECTED}

If demyelinating etiologies for the cranial nerve deficits are considered, then the postgadolinium scans should be extended to cover the whole brain. If one identifies lesions that enhance and lesions that do not, it would imply a polyphasic disease such as multiple sclerosis.

1. Repeat sequence 6 according to Table A7.4.7. Change slice thickness to $5 \mathrm{~mm}$ to cover the entire brain.

2. Repeat sequence 7 according to Table A7.4.8. Change slice thickness to $5 \mathrm{~mm}$ in order to cover the entire brain. 


\section{COMMENTARY}

\section{Background Information}

The vagus nerve is the longest-running of the cranial nerves. Its recurrent laryngeal nerve branch loops under the aortic arch on the left and the subclavian artery on the right. Therefore, in the evaluation of vocal cord paralysis, the vagus nerve's origin from the medulla, its egress through the pars vasculosa of the jugular foramen, its course within the carotid sheath to the take-off of these vessels, and the course of the recurrent laryngeal nerve looping under the subclavian artery (right) or aorta (left) and ascending in the tracheoesophageal groove, must be examined.

If there are multiple cranial nerve deficits or bilateral hearing deficits, one might be dealing with neurofibromatosis type II, subarachnoid seeding on nerve roots, sarcoidosis, Lyme disease, granulomatous infections, etc., all of which require greater scrutiny of the post-contrast scans. The whole brain might need to be evaluated for a source of drop metastases.

\section{Critical Parameters}

The problems associated with jugular flow artifacts are the biggest hurdle in creating diagnostic images in this regions. Too often the turbulence causes soft tissue signal in the jugular foramen, and the slow flow seen as "enhancement" on the post-contrast studies only complicates matters. Good quality $T_{1}$-weighted scans may be the lifesaver. As for the vagus nerve, the issues related to this evaluation revolve around on how low the scanning must go. Often the chest images are distorted due to respiratory and/or pulsation artifact.

\section{Troubleshooting}

Again, MR venograms will clarify most issues as they relate to the jugular foramen. If one is able to obtain a history of palatal palsy, uvular deviation, or other pharyngeal symptomatology, it may point to an upper vagus nerve lesion, and the need to extend the scan into the lower neck and/or chest may be obviated. To get good-quality images of the left recurrent laryngeal nerve at the aortic arch, a chest protocol with respiratory gating may be required. If this is a consideration, a dedicated chest MRI may be in order, and techniques will have to be employed such as cardiac gating and respiratory compensation/triggering. In order to accentuate the visualization of the laryngeal nerve at the aortic arch, one of the best pulse sequences to utilize would be a "double inversion recovery" better known as black blood imaging (see Table A7.4.10). This technique allows the user to obtain a "black blood" image (which is fantastic for vascular anatomy) while maintaining a parenchymal fat plane. This fat plane enables the radiologist to essentially roadmap the nerve from origin-to-pathology. We like to think of this "black blood" technique as a snapshot image that acquires sequential slices in a breath hold and cardiac gated style. When utilizing this pulse sequence, ensure that the technique is done prior to the administration of contrast medium. Post-contrasted black blood imaging will often leave the user with less than desirable results.

\section{Anticipated Results}

One should be able to see cranial nerves IX and $\mathrm{X}$ coursing together from the medulla to the jugular foramen. The spinal accessory nerve is virtually never separately discerned, and the hypoglossal nerve is inconstantly visualized. Schwannomas of these nerves will be evident on post-contrast studies.

\section{Time Considerations}

In chasing vagus nerve lesions, one may be forced to employ a head, neck, and chest coil. The time demands on switching the coils and repositioning the patient may be prohibitive, and the case should be charged accordingly. In most instances the post-contrast transverse scans are the most valuable, so reducing the NEX or employing fast spin echo $T_{1}$-weighted scans may be economical.

\section{Index of Terms}

The clinical imaging instructions and terminology utilized in this unit are primarily geared towards General Electric equipment. An Index of Terms is provided that should bridge the gap of vendor specific terminology.

Chemical saturation A technique that applies an additional radiofrequency (RF) pulse (at a desired distance from the center frequency) to selectively suppress a tissue. This technique can be utilized to suppress the signal from water, fat, or silicone.

Echo time $\left(\boldsymbol{T}_{\mathrm{E}}\right)$ The time that is measured from the initiation of the initial RF (radio frequency) pulse and the peak of the echo.

Echo train length (ETL) In fast spin echo or turbo spin echo imaging, the ETL will actually equal the number of echoes prescribed per $T_{\mathrm{R}}$. Successive $180^{\circ}$ refocusing pulses are ap- 


\begin{tabular}{|c|c|}
\hline Patient position & Supine \\
\hline Scan type & Fast spin echo \\
\hline Imaging plane (orientation) & Transverse \\
\hline Central slice or volume center & Chest \\
\hline Echo time $\left(T_{\mathrm{E}}\right)$ & $30-60 \mathrm{msec}$ \\
\hline Receiver bandwidth (RBW) & $32 \mathrm{kHz}$ \\
\hline Echo train length (ETL) & 32 \\
\hline Repeat time $\left(T_{\mathrm{R}}\right)$ & Minimum \\
\hline Inversion time $\left(T_{\mathrm{I}}\right)$ & Auto $(\sim 600 \mathrm{msec})$ \\
\hline Flip angle (FA) & $180^{\circ}$ \\
\hline Fields of view $\left(\mathrm{FOV}_{\mathrm{x}}, \mathrm{FOV}_{\mathrm{y}}\right)$ & $240 \mathrm{~mm}, 240 \mathrm{~mm}$ \\
\hline Resolution $(\Delta x, \Delta y)$ & $0.94 \mathrm{~mm}, 1.25 \mathrm{~mm}$ \\
\hline Number of data points collected $\left(N_{\mathrm{x}}, N_{\mathrm{y}}\right)$ & 256,192 \\
\hline Slice thickness $(\Delta z)$ & $5 \mathrm{~mm}$ \\
\hline Number of slices & Variable \\
\hline Slice gap & $2 \mathrm{~mm}$ \\
\hline Number of excitations (NEX) & 1 \\
\hline ZIP 512 & $\begin{array}{l}\text { Yes (changes resolution to } 0.47 \\
\mathrm{~mm}, 0.63 \mathrm{~mm} \text { ) }\end{array}$ \\
\hline Slice series & Interleaved \\
\hline ECG gating & Yes \\
\hline Scan time & $\sim 5-6 \min$ \\
\hline \multicolumn{2}{|c|}{$\begin{array}{l}{ }^{a} \text { For this sequence, cardiac gating is essential. The minimum } T_{\mathrm{R}} \text { to be utilized will be calculated by } \\
\text { the imaging system. Typically, the image may look proton density weighted because the } T_{\mathrm{R}} \mathrm{s} \text { are } \\
\text { relatively long ( } 1500 \mathrm{msec} \text { to } 1800 \mathrm{msec} \text { ) and the } T_{\mathrm{E}} \mathrm{s} \text { are generally short ( } 30 \mathrm{msec} \text { to } 60 \mathrm{msec} \text { ). The } \\
\text { inversion time is typically set by the MR system. The system calculates the "inversion time" needed } \\
\text { to suppress the overall signal from blood based on the heart rate. This inversion time will change slightly } \\
\text { from patient to patient. A neurovascular coil is used in this sequence when the imaging position is neck } \\
\text { or above. A torse array coil is used when the imaging position is chest. }\end{array}$} \\
\hline
\end{tabular}

plied to "rephase the dephasing" protons in an effort to maximize the number of lines of $k$ space per $T_{\mathrm{R}}$. The formula for scan time in relation to Fast Spin Echo imaging and ETL is as follows:

Scan time $=\left(T_{\mathrm{R}}\right) \times($ no. of phase

encoding steps/ETL $) \times(\mathrm{NEX})$

Extended dynamic range (EDR) An imaging-enhancement tool that will allow the utilization of 32-bit data processing as opposed to the standard 16-bit processor. EDR in this way should improve SNR and resolution, but will utilize twice as much memory as a conventional acquisition.

Flow compensation Sometimes more widely expressed as gradient moment nulling, this is a way in which the system places flowing or moving spins into "phase coherence" with stationary spins.

Inversion time $\left(T_{\mathrm{I}}\right)$ With inversion recovery pulse sequences, typically the inversion time is the time from the first $180^{\circ} \mathrm{RF}$ pulse to the center of the next $90^{\circ} \mathrm{RF}$ pulse. This inver- sion time will essentially "null" the desired tissue depending on how long or short the $T_{\mathrm{I}}$ (inversion time) selected is, and the $T_{1}$ relaxation time of the corresponding tissue.

Multi-phase is an imaging option that allows the user to run a pulse sequence over and over again. Its application is primarily utilized in contrast-enhanced angiography when attempting to "catch" an arterial phase of contrast media, followed by a mixed arterial and venous filling phase. Multi-phase allows continual scanning of a desired area independent of the reconstruction phase.

No phase wrap (NPW) will prevent wrap around artifacts (also known as aliasing artifacts) in the phase encoding direction. NPW should only be used when necessary because of the following:

a. NPW doubles the FOV in the phase direction.

b. NPW essentially doubles the phase encoding steps (to maintain resolution).

c. With NPW, you must reduce the NEX by half in order to maintain scan time. 
Number of excitations (NEX) is a factor that is utilized to calculate the overall scan time and will directly effect the SNR. NEX is essentially the number of times that data are sampled per acquisition. Note that increasing the NEX to achieve overall better SNR is a rather inefficient way to improve signal. Doubling the NEX from 2 to 4 will only yield a $40 \%$ increase in SNR while it doubles scan time. Take note of the formula for scan time:

Scan time $=\left(T_{\mathrm{R}}\right) \times($ no. of phase

encoding steps $) \times(\mathrm{NEX})$

Receiver bandwidth (RBW) The range of frequencies that the MRI scanner is actually "tuned" to receive. This will directly affect the overall SNR. This will not be done by increasing or decreasing the signal, but rather there will be an increase and/or decrease in the amount of noise received relative to the alteration of the RBW. An increase in the RBW will increase the range of frequencies that the scanner will evaluate and thus decrease the overall SNR. In comparison, utilizing a narrow bandwidth should yield less noise and improve the overall SNR. The relationship of the receiver bandwidth and SNR can be thought of as inversely proportional to the square root of the bandwidth.

Rectangular field of view (REC FOV) Asymmetric field of view (typically in the phase encoding direction). REC FOV is typically utilized when a body part is longer in one direction than another. By utilizing an asymmetric FOV, the system will not collect a portion of the data, thereby decreasing the scanning time.

Repetition time $\left(\boldsymbol{T}_{\mathbf{R}}\right)$ The time in a pulse sequence between successive excitation pulses.

Spatial saturation employs an additional RF pulse to cause moving spins within a determined area to be selectively dephased. This application will reduce motion from flow and/or respiratory artifacts and will limit the number of slices per $T_{\mathrm{R}}$ in general. Since a spatial saturation pulse employs additional RF, which is to be deposited into the patient, special attention should be focused on the SAR (specific absorption rate). Today all MRI scanners have a program that internally monitors how much RF can be applied over a given period of time. This formula takes into account the patient's body weight. This actual body weight needs to be accurately input at all times for patient safety. An inappropriate weight will cause the improper limit of RF to be transmitted relative to a safe period of time in which this is to occur.

Tailored RF An imaging option that improves image quality on fast spin echo (FSE) sequences with relatively short $T_{\mathrm{E}}$ 's. Tailored RF will improve edge blurring by reducing overall echo spacing.

ZIP 512/ZIP 1024 Better known as "zerofill interpolation process," this is a reconstruction algorithm that allows the user to scan at a $256 \times 256$ matrix and then the data are zerofilled to a $512 \times 512$ matrix (or $1024 \times 1024$ respectively).

ZIP 2/ZIP 4 Slice ZIP essentially is also a "zerofill interpolation process" that will create additional slices through the interpolation procedure. These slices are created with an offset of $50 \%$ of the original imaging slice locations.

\section{Literature Cited}

Castillo, M. and Mukherji, S.K. 1996. Magnetic resonance imaging of cranial nerves IX, X, XI, and XII. Top. Magn. Reson. Imaging 8:180-186.

Chong, V.F. and Fan, Y.F. 1996. Jugular foramen involvement in nasopharyngeal carcinoma. $J$. Laryngol. Otol. 110:987-90.

Karpati, R.L., Loevner, L.A., Cunning, D.M., Yousem, D.M., Li, S., and Weber, R.S. 1998. Synchronous hypoglossal nerve and sympathetic nervous system plexus schwannomas. AJR Am. J. Roentgenol. 171(6):1505-1507.

Ortiz, O. and Reed, L. 1995. Spinal accessory nerve schwannoma involving the jugular foramen. AJNR Am. J. Neuroradiol. 16:986-989.

Pribitkin, E.A., Rondinella, L., Rosenberg, S., and Yousem, D.M. 1994. Superficial siderosis of the central nervous system: An underdiagnosed cause of sensorineural hearing loss and ataxia. Am. J. Otol. 15:415-418.

Rubinstein, D., Burton, B.S., and Walker, A.L. 1995. The anatomy of the inferior petrosal sinus, glossopharyngeal nerve, vagus nerve, and accessory nerve in the jugular foramen. AJNR Am. J. Neuroradiol. 16:185-194.

Russo, C.P., Smoker, W.R., and Weissman, J.L. 1997. MR appearance of trigeminal and hypoglossal motor denervation. AJNR Am. J. Neuroradiol. 18:1375-1383.

Shellock, F.G. 1996. Pocket Guide to MR Procedures and Metallic Objects. Lippincott-Raven, Philadelphia.

Thompson, E.O. and Smoker, W.R. 1994. Hypoglossal nerve palsy: a segmental approach. $R a-$ diographics 14:939-958.

Contributed by Robert W. Evers and David M. Yousem

The Johns Hopkins Hospital

Baltimore, Maryland
Head and Neck

A7.4.11 